\title{
Orientation System based on Speculative Computation and Trajectory Mining
}

\author{
João Ramos ${ }^{1 \star}$, Tiago Oliveira ${ }^{1 \star \star}$, Ken Satoh ${ }^{2}$, José Neves $^{1}$, and Paulo Novais ${ }^{1}$ \\ 1 Algoritmi Centre, Department of Informatics \\ University of Minho \\ Braga, Portugal \\ \{jramos, toliveira, jneves, pjon\}@di.uminho.pt \\ 2 National Institute of Informatics \\ Hitotsubashi, Chyoda-ku \\ Tokyo, Japan \\ ksatoh@nii.ac.jp
}

\begin{abstract}
Assistive technologies help users with disabilities (physical, sensory, intellectual) to perform tasks that were difficult or impossible to execute. Thus, the user autonomy is increased through this technology. Although some adaptation of the user might be needed, the effort should be minimum in order to use devices that convey assistive functionalities. In cognitive disabilities a common diminished capacity is orientation, which is crucial for the autonomy of an individual. There are several research works that tackle this problem, however they are essentially concerned with user guidance and application interface (display of information). The work presented herein aims to overcome these systems through a framework of Speculative Computation, which adds a prediction feature for the next move of the user. With an anticipation feature and a trajectory mining module the user is guided through a preferred path receiving anticipated alerts before a possible shift in the wrong direction.
\end{abstract}

\section{Introduction}

Cognitive disability is a broad concept which includes different intellectual or cognitive deficits. These deficits may be present from birth (like birth defect) or may be acquired later (like traumatic brain injury). More precisely, this term is used to define a person who has more difficulties in one or more types of mental tasks when compared to an ordinary person [17]. A disability may be present in several levels of incidence, varying from mild to extreme. An individual with

* This work has been supported by COMPETE: POCI-01-0145-FEDER-007043 and FCT Fundação para a Ciência e Tecnologia within the Project Scope: UID/CEC/00319/2013. The work of João Ramos is supported by a doctoral the FCT grant SFRH/BD/89530/2012.

** The work of Tiago Oliveira is also supported by the FCT grant with the reference SFRH/BD/85291/2012 
severe or extreme cognitive disabilities needs constant assistance throughout his everyday life whereas a mild to moderate disabled person may be capable of having an independent life, only requiring some assistance in certain activities.

Assistive technology aims to increase, maintain or even improve functional capabilities of a person with disabilities [1]. A mental task commonly affected is orientation, which is imperative for an independent life. Thus, it is necessary to have technologies that assist the user during his travel between home and office/school. Using an orientation device the user is sufficiently autonomous to travel between his current location to a predefined destination. Current approaches focus essentially on the guidance activity, giving more attention to the information display and to the communication with a caregiver $[5,11,9]$.

This work proposes an orientation system that, besides guiding the user, tries to anticipate possible mistakes. An alert is triggered when the user is expected to make a wrong turn in his path. In order to adapt the system to the user it is also included an trajectory mining feature so it is possible to calculate a path that is preferred by the user (which may not be the shortest one).

This paper is organized as follows. Section 2 briefly presents related work concerning orientation systems for people with cognitive disabilities. Section 3 provides a description of the orientation system giving emphasis to new developments on the Speculative Module and Trajectory Mining Module. The Speculative Module that hosts the framework for Speculative Computation is explained in Section 4. On Section 5 the Trajectory Module used to get a path according to the user's preferences is described. Finally, conclusions are drawn and future work considerations are made in Section 6.

\section{Related Work}

The works described in [11], [5] and [9] are examples of three different orientation methods for people with cognitive disabilities. In these examples the main goal is to guide a person outdoors from the current location to a predefined destination. The difference between them resides in how this is done, but all are particularly focused on the user interface. Thus, they lack the predictive capabilities which would allow them to anticipate wrong user actions and apply necessary measures to avoid them, and the capability of adjusting the path to user preferences.

The technological development of smartphones brought more portability to the user since it became possible to execute applications in small and portable devices. These devices are specially important to people with cognitive disabilities since it is through them that the user may contact his caregiver. In order to execute an application to guide the user, the developers have to pay special attention to the interface [8].

With the goal of guiding cognitive disabled people and considering the systems interface, Carmien et al. [5] developed an application that enables the user to travel using a public transportation system reducing the effort needed to understand complex transportation maps. While the user is travelling, a personal 
travel assistant ensures that the user has taken the correct bus, alerting him otherwise.

Liu et al. [11] focused their research on the display of instructions. They used static pictures with overlaid arrows (or highlighted areas in an image), audio and/or visual messages to guide the user. Their objective was finding the best way of providing the directions to the user using either static pictures reflecting user perspectives with landmarks that are easy to find or visual/audio messages whenever an image is not available.

A different approach was used by Fraunhofer Portugal in the AlzNav orientation system [9]. This system used an arrow that resembles a compass to guide the user. Thus, the user has to interpret the information presented on the device's screen. Besides the compass, the user has also information about the street he is and the distance he should travel in the calculated direction. This systems has also a monitoring system enabling caregivers to know the current position of the user through a SMS.

The previously presented approaches tackle some important aspects of orientation systems for people with cognitive disabilities. However there are some features that should be considered in order to make the system adaptable to the user and not the other way around. Predicting user steps is a big advantage for this type of system since it is possible to identify critical points in a certain path and alert the user before he makes a mistake. If the system is able to predict when an error will occur, it can issue an alert to the user reinforcing the right path. Another important feature is the ability to adjust the path to the user since he may prefer to travel for a longer but preferred path instead of taking the shortest one. These are the kind of features proposed in this work. The goal is to develop an orientation system that adapts to the user, maximizing his autonomy and consequently his independence.

\section{System Description}

CogHelper is an ongoing project $[16,14,15]$ with two main goals: provide an efficient orientation system for people with cognitive disabilities and provide a tracking system for caregivers. The former is accomplished through an augmented reality interface so users only need to align the mobile device with the correct travelling path in order to see a green arrow indicating the right direction. The latter enables caregivers to know in any time the current position of the person with disabilities.

The orientation method under development is conceived for outdoors which has been fully described in [13]. The core of the system is considered to be fully developed, i.e., both applications for caregivers (mobile and web), the webservices (running on the server) needed in order to assure communications between applications, the database, and finally the mobile application for the person with cognitive disabilities. At this point this application uses augmented reality for the orientation and the selected path is the shortest one (not being adapted to the user as proposed in this paper). 
The primary target of CogHelper system is people with cognitive disabilities. Thus the mobile application intended to this audience is composed by four layers (see Fig. 1), each with specific functions. The Information Layer stores the information for the normal execution of the application (like user data and his contacts). In the Localization Layer the current user position is retrieved from the GPS module of the device (or from the network) and is used by the Navigation Algorithm. To this information is added data gathered from the device sensors, like the camera, magnetic sensor and accelerometer (enabling the system to compute the device's direction), which are used by the decision algorithm to ensure the user is travelling in the correct path. All information is then presented to the user through the user interface (under the System input/output). A detailed description of previously cited modules is done in [14]. Being an ongoing project, CogHelper is being improved with new modules in order to give the system an adaptability feature. The Trajectory mining and Speculative Module components (depicted in Fig. 1) are responsible for the adaptability of the system to the user.

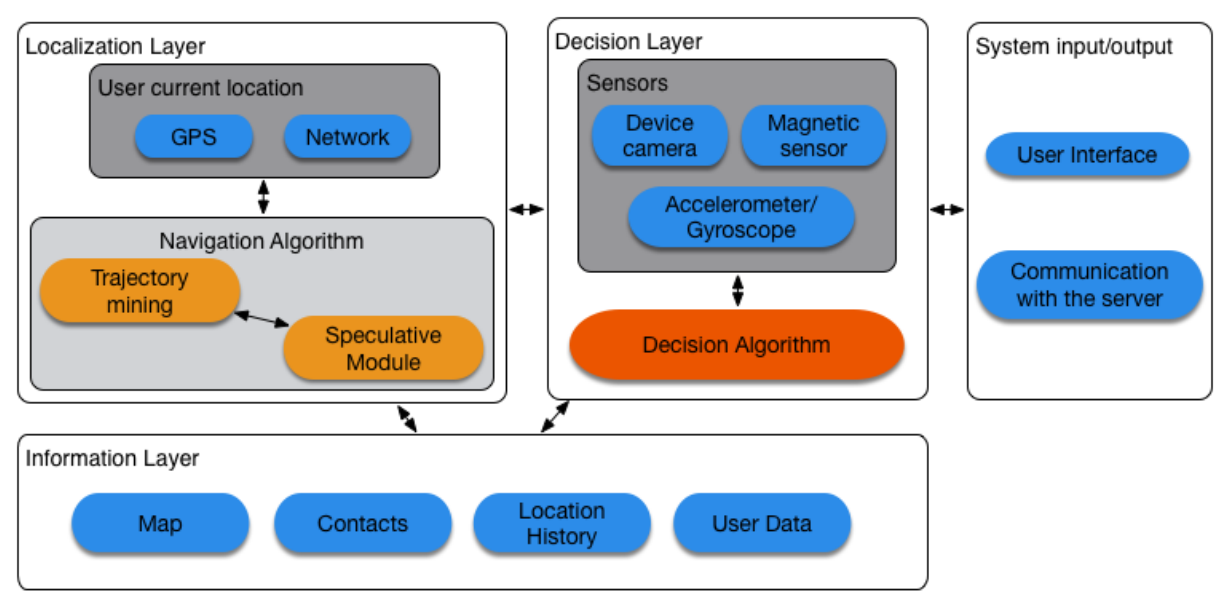

Fig. 1: Information layers of the CogHelper Module for People with Cognitive Disabilities

The Trajectory mining component generates a path that is preferred by the user (which may not be the shortest one), i.e., the path is calculated according to the preferences of the user (historic data from previous uses of the application). The Speculative Module ensures that the user is travelling in the correct path alerting him otherwise. These modules are described in more detail in Section 5 and Section 4, respectively.

Moreover, one cannot exclude the development of additional features, such as the ones described in [6], in order to detect other user activities, namely fall detection. 


\section{Speculative Module for Users with Cognitive Disabilities}

The Speculative Module under the mobile application for people with cognitive disabilities has the objective of predicting the next step of the user (when using the orientation system) and use that prediction to set the information that should be displayed to the user (alert/warning or acknowledge messages). The execution of the Speculative Computation resembles an interface between the rules with the instructions for the correct path, the set of default values (predictions about user travels from one location to another), and real information returned by information sources (informing the real journey of the user).

Through the use of this module the system continues its execution using a default value (whenever the real information is missing) or using the real one (returned from the information sources). Thus, the system does not enter an idle state when there is missing information. It tries to generate a tentative solution for the problem, which is revised when the real information is received (to verify if the default value is consistent with the real one).

For the execution of the Speculative Computation module the computation changes between its normal execution phase (Process Reduction Phase) and temporarily to a revision phase (Fact Arrival Phase) to revise the computation according to the received values. The initial information, before execution, represented in the Speculative Computation framework includes:

1. All the possible paths between two points, in the form of connections between intermediary points, as facts in the knowledge base;

2. The transitions between points usually performed by the user as default values;

3. Information of whether a point is included or not in the recommended path as default values;

4. A set of rules that structure the derivation of the path the user is likely to follow given the information during execution and the issuing of alerts/warnings in case of a potential mistake;

At the beginning of the computation, when there is no information regarding the actual position of the user and his transitions between the most relevant points, the defaults are used in the Process Reduction Phase to build the most likely path, step by step, and issue the warnings for potential mistakes or acknowledgements of correctly taken steps. A warning is issued whenever a user is likely to take the wrong path, which may happen when the defaults tell the computation that the user will make a transition to a point not included in the correct path. Through Fact Arrival, the GPS sensor and a recognizer inform the Speculative Module of the actual transitions of the user and whether the points are indeed part of the correct path or not. If the user actually moves to a point not included in the correct path, the recognizer re-calculates the path to the destination and a point previously out of the correct path may suddenly become part of the new path. Fact Arrival Phase is the mechanism through which this 
information is updated and the tentative paths produced for a user are adjusted and improved.

Items 1, 2, and 3 from the list above are obtained from a Trajectory Mining Module. Item 1 corresponds to the calculation of the possible paths between two points, producing a reduced graph, with only the most relevant points an the connections between them. Item 2 is obtained from the pattern mining of the trajectories usually taken by the user, reflecting his walking habits. Finally, item 3 corresponds to the calculation of the recommended path between the point of origin and the destination, expressed in the form of intermediary points included in the route. It is also stated which points are not included in this route. As an example, one can consider the graph of Figure 2, in which the objective is for the user to move from node 1 to node 3. From the Trajectory Mining Module it is possible to know that the user usually moves from 1 to 2 , from 2 to 3 , but also from 3 to 4 , from 4 to 5 , from 5 to 6 , and from 6 to 3 . Additionally, it is possible to determine that nodes 1,2 , and 3 are part of the shortest path, while node 4 is obviously not. However, since the user, when in node 2 , usually moves to 4 , this is identified during Process Reduction as a point where a mistake may happen and, as a result, a warning is issued. If, during Fact Arrival, it is confirmed the user indeed moves to 4 , this node becomes now part of the route and the alternative path including 5 and 6 is selected. Although simple, this example illustrates the role of the Speculative Module in producing instructions for the user and preventing mistakes in his path.

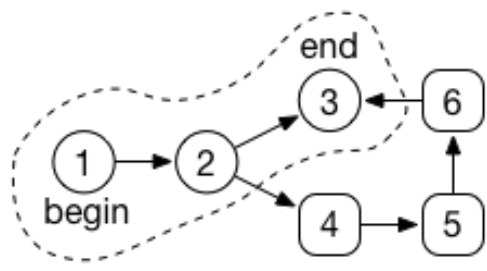

Fig. 2: Possible ways to travel between locations 1 and 3.

Below there is a logic program that represents the formalization of the problem depicted in Figure 2 and the situation described above according to the Speculative Computation Framework for Users with Cognitive Disabilities. Its components include: $\Sigma$, a representation of existing system modules responsible for providing information; $\mathcal{E}$, the predicates which represent the necessary information to derive the path of the user; $\Delta$ is the default answer set and consists in a set of default values; $\mathcal{A}$, a set of abducible predicates; and $\mathcal{P}$ is a logic program with a set of clauses. In the logic program given below the literal path $(a, b)$ denotes that there is a physical connection between the locations $a$ and $b$, thus the user may travel between them. The literal show_next_point is used to indicate that the system must show the next location to which the user should travel. This location may be an intermediate point or the final destination. Whenever 
the user travels in the wrong direction the literal show_user_warning is activated indicating to the system that it must alert the user. In the set $\mathcal{E}$ there are the predicates user_travel $(a, b)$ (which states that the user will travel from location $a$ to location $b$ ) and included(a) (to indicate if a location $a$ is part of the route). The values for these predicates are asked from the information sources gps_sensor and recognizer, respectively. The former verifies if the user is travelling from point $\mathrm{A}$ to $\mathrm{B}$. The latter checks if point $\mathrm{B}$ is included in the set of valid locations.

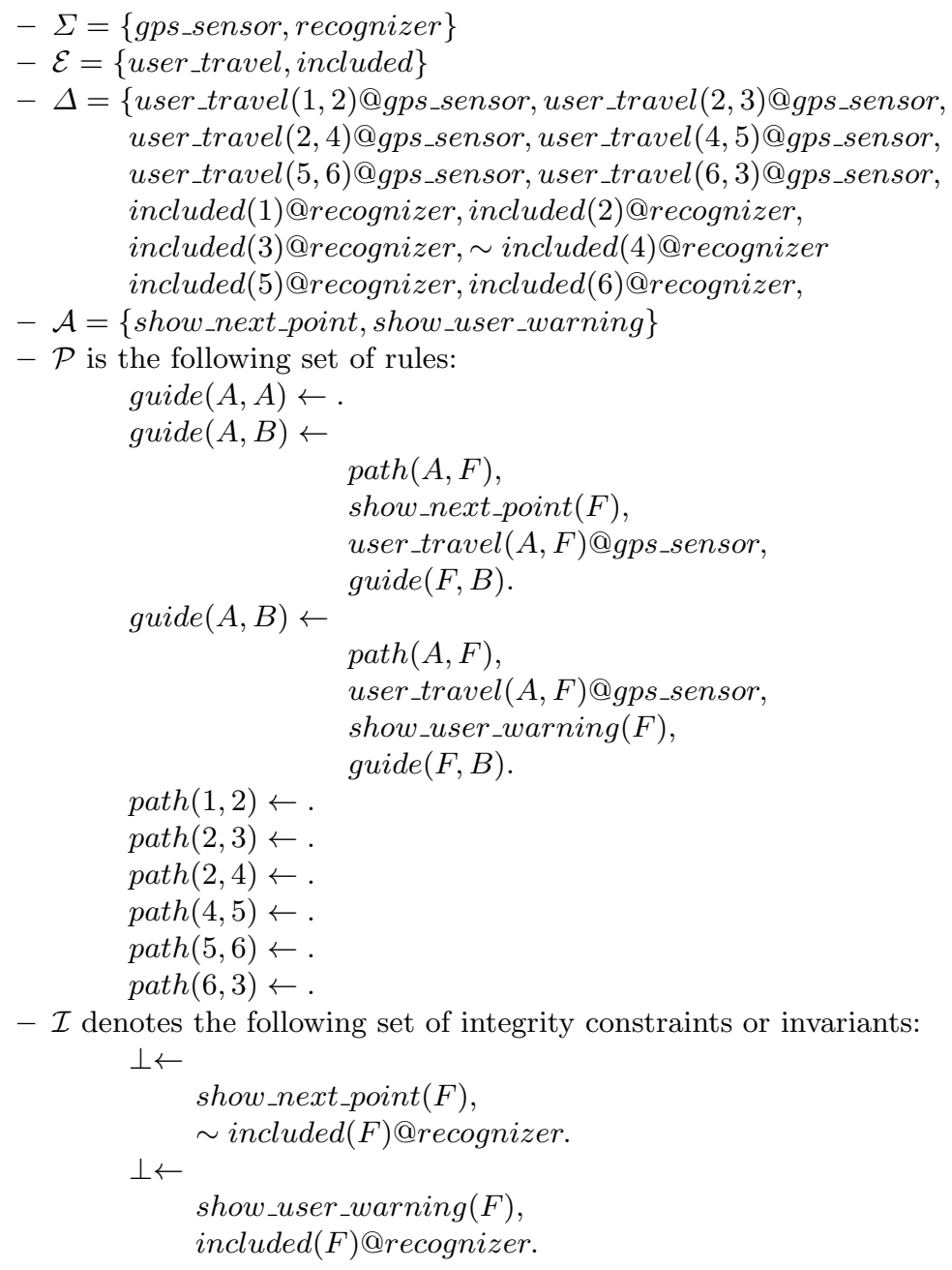

\section{Trajectory Mining Module}

Advances in mobile computation (e.g., smartphones) and in location-acquisition methods (e.g., GPS module) enabled the gathering of massive spatial trajectory 
data, which, in turn, has raised the interest of researchers in trajectory data mining [19]. According to [7] there are three important attributes when considering Behavioural Pattern Mining: location, trajectory and behaviour. The first attribute considers the extraction of important user locations (like home or office). The second one considers the trajectory modeling through the extraction of regular routes. The last attribute emphasizes the extraction of behavioural patterns. Thus the system may be able to predict the user's destination through his current path.

Through active recording (the user location is logged only when the application is running) it is possible to obtain the position of the user. However there are a few steps that precede the trajectory pattern mining like trajectory data preprocessing and trajectory data management [19].

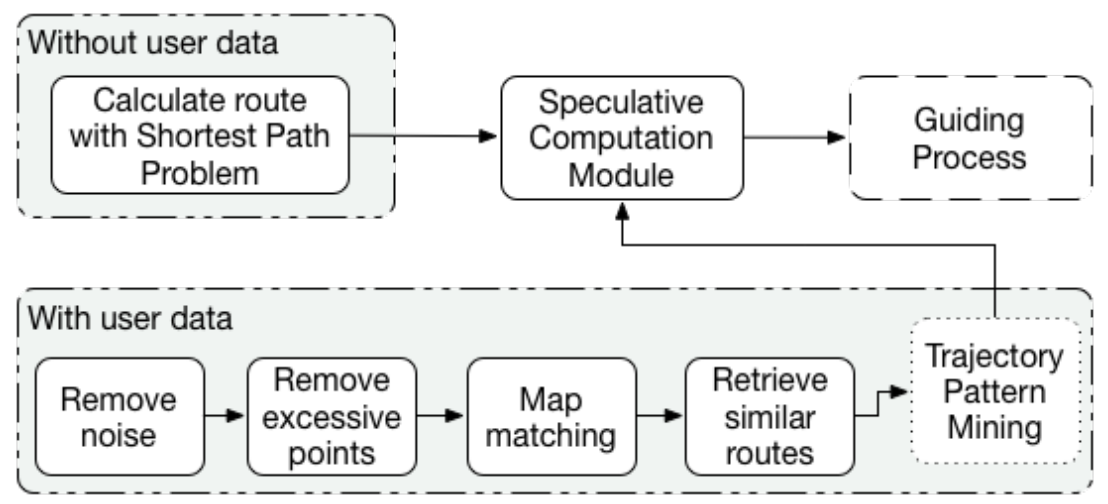

Fig. 3: Generation of default values schema

For the mining module operation it is important to consider the existence of user historic data. When the user is using CogHelper for the first time (thus, there isn't any information about his travelling habits) the system calculates the shortest path and uses it as input for the speculative computation module in order to guide the user. When there is available information about the user, the mining module extracts similar routes according to the current user location and the intended destination and use them for the trajectory pattern mining. Ending this process the data is sent to the speculative computation module (as default values) so the system is able to guide the user. Before applying the pattern mining the retrieved data need to be preprocessed. An illustration of this process is presented in Figure 3.

\subsection{Data Preprocessing}

In a first step (trajectory data preprocessing) it is important to remove the noise from each collected position (Figure 4a is an example of raw data collected during 
a travel). There may be points that may appear outside the travelled route (due to a bigger GPS error), which should not be considered for the trajectory data mining (Figure 4b). Then, since collected locations represent a large number of samples, it is important to remove excessive data, i.e., points that do not bring useful information like intermediate points in roads without intersections.

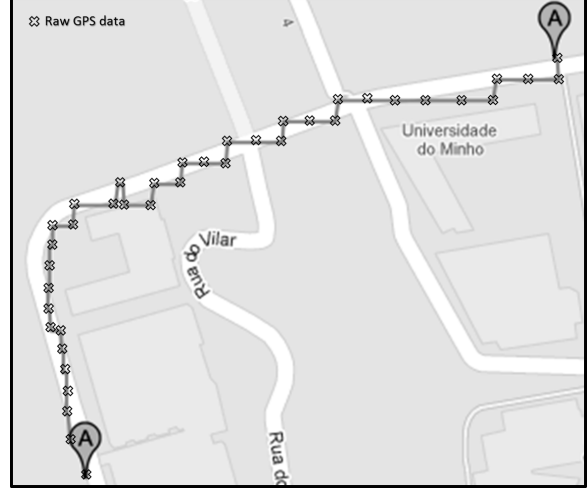

(a) Route obtained from database raw data

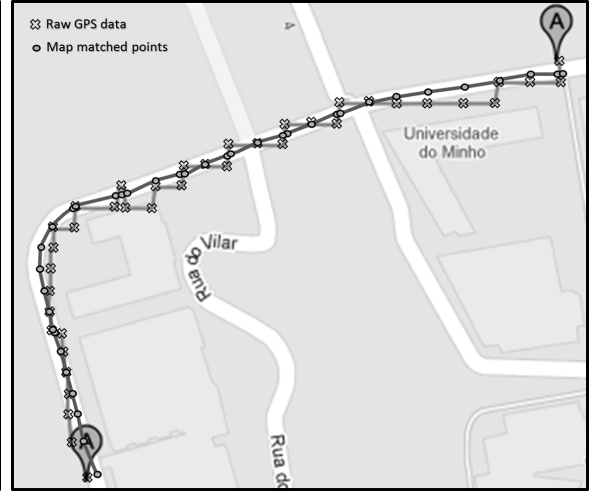

(b) Error removal from raw data

Fig. 4: Trajectory data processing

After this process, the remaining data should represent locations on a map that may appear slightly outside the road. Thus, a map matching process is necessary in order to align the collected data with the existing real maps.

Ending this preprocessing stage and before starting the trajectory data mining process, the system has to obtain similar routes (e.g., travel paths with the same destination and a similar starting point). Thus, instead of using all routes for this stage, the system selects the similar routes (or similar route parts) through similarity/distance functions. When, for a user, there is no route for an intended destination the system selects the shortest path.

\subsection{Trajectory Mining}

Finally, the trajectory data mining may begin. For this process there are different types of pattern mining. The described process may be considered as a standard for the use of trajectory data mining, however there might be some derivations of it. According to its own proposes an author may adapt this standard for his algorithm or system.

In [10] the authors consider a trajectory pattern as a set of individual trajectories which share an identical sequence of visited places. For their trajectory pattern mining in an initial stage the authors try to get a set of regions of interest (which is possible by different approaches) and then the authors try to define 
the trajectory patterns. Giannotti et al. intend to apply their trajectory pattern mining in the analysis of traffic flows. An extension of this work is presented in [12] in which the authors make use of all trajectories saved on the database to construct a predictive model in order to be able to predict the user's (or object's) next move (e.g., predict where the user will be when the GPS module is temporarily unavailable).

A different approach is applied in [2]. Here the aim is to automatically obtain the frequent moves, neglecting the time at which their occur. To the authors a trajectory is an ordered list of stops and moves, i.e., the user moves between to places (considered as stops) in which he stays for a given time interval. For their purpose the authors intend to discover the pattern of moves between two places usually done by the users regardless the intermediate points (e.g., the streets or roads used). Using this data Alvares et al. could obtain answers for questions like the most frequent stops during a period of time, which stops have a duration higher than a predefined threshold, among others.

Chen, in [7], propose a model in which after the geo-coordinates extraction, a tree-based hierarchy graph is built. Through this the author intends to apply hierarchy density clustering algorithms like OPTICS [18] to find patterns in the recorded data. The OPTICS algorithm [18] has been used for mining people's life pattern using GPS log data. This is an algorithm that tries to find densitybased clusters in the spatial data [4]. For this process a distance metric between location points is required in order to group the data into clusters.

Considering the different models and their application our goal is to define the best strategy to apply in CogHelper. According to our research and considering the goal for each method/algorithm, we consider that OPTICS will be the most appropriated for our system.

\section{Conclusions and Future Work}

This work proposes a trajectory mining method which produces a set of default values. These values are considered the predictions for the directions that the user should follow in order to travel between his current location to the intended destination. The trajectory mining module process uses data from previous executions of the system, which are used to obtain the best travelling path (according to user preferences) and critical points, such as intersections in which the user may take the wrong turn. Through this set of default values the Speculative Computation module is used as a mechanism that determines if it is necessary to issue an alert or not. The integration of these two modules are the main contribution of this work. The speculative framework is independent of how the trajectory mining is achieved using the calculated values to ensure the correct travel. A structured reasoning method is provided through the combination of these modules. After preprocessing the data, it is possible to apply different trajectory mining techniques. Our future goal is to determine the most appropriate one for the problem. 
In order to better perceive the advantages and disadvantages of CogHelper, a comparison with different systems (presented in Section 2) should be conducted. However this may not be an easy task since CogHelper has an adaptability feature that is not present in other systems. These last are mainly focused on the user interface (on how the information should be available to the user) and do not adapt the travelling path to user preferences.

As future work, user privacy issues will be considered taking into account the work developed in [3].

\section{References}

1. Alper, S., Raharinirina, S.: Assisitive Technology for Individuals with Disabilities: A Review and Synthesis of the Literature. Journal of Special Education Technology 21(2), 47-64 (2006)

2. Alvares, L.O., Bogorny, V., Antonio, J., Fernandes De Macedo, J.A., Moelans, B., Spaccapietra, S.: Dynamic Modeling of Trajectory Patterns using Data Minig and Reverse Engineering. ER 2007 pp. 149-154 (2007)

3. Andrade, F., Neves, J., Novais, P., Machado, J., Abelha, A.: Legal security and credibility in agent based virtual enterprises. In: IFIP Advances in Information and Communication Technology. vol. 186, pp. 503-512. Springer New York LLC (2005)

4. Ankerst, M., Breunig, M.M., Kriegel, H.P., Sander, J.: Optics: Ordering points to identify the clustering structure. ACM Sigmod Record pp. 49-60 (1999)

5. Carmien, S., Dawe, M., Fischer, G., Gorman, A., Kintsch, A., Sullivan, J.F.: Sociotechnical environments supporting people with cognitive disabilities using public transportation. ACM Transactions on Computer-Human Interaction 12(2), 233$262(2005)$

6. Castillo, J.C., Carneiro, D., Serrano-Cuerda, J., Novais, P., Fernández-Caballero, A., Neves, J.: A multi-modal approach for activity classification and fall detection. International Journal of Systems Science 45(4), 810-824 (2014)

7. Chen, Z.: Mining individual behavior pattern based on significant locations and spatial trajectories. In: 2012 IEEE International Conference on Pervasive Computing and Communications Workshops, PERCOM Workshops 2012. pp. 540-541 (2012)

8. Dawe, M.: Desperately seeking simplicity: how young adults with cognitive disabilities and their families adopt assistive technologies. In: Proceedings of the SIGCHI conference on Human Factors in computing systems. pp. 1143-1152. CHI '06, ACM (2006)

9. Fraunhofer Portugal: AlzNav (2012), http://www.fraunhofer.pt/en/ fraunhofer $\left\{\backslash\right.$ \}aicos/projects/internal $\left\{\backslash \_\right\}$research/alznav.html

10. Giannotti, F., Nanni, M., Pinelli, F., Pedreschi, D.: Trajectory Pattern Mining. In: Proceedings of the 13th ACM SIGKDD International Conference on Knowledge Discovery and Data Mining. pp. 330-339. KDD '07, ACM (2007)

11. Liu, A.L., Hile, H., Borriello, G., Kautz, H., Brown, P.A., Harniss, M., Johnson, K.: Informing the Design of an Automated Wayfinding System for Individuals with Cognitive Impairments. In: Proceedings of Pervasive Health '09. vol. 9, p. 8 (2009)

12. Monreale, A., Pinelli, F., Trasarti, R.: WhereNext : a Location Predictor on Trajectory Pattern Mining. In: Proceedings of the 15th ACM SIGKDD international conference on Knowledge discovery and data mining - KDD '09. pp. 637-645 (2009) 
13. Ramos, J., Costa, A., Novais, P., Neves, J.: Interactive Guiding and Localization Platform. International Journal of Artificial Intelligence (IJAI) 12(1), 63-78 (2014)

14. Ramos, J., Novais, P., Satoh, K., Oliveira, T., Neves, J.: Speculative Orientation and Tracking System. International Journal of Artificial Intelligence (IJAI) 13(1) (2015)

15. Ramos, J., Oliveira, T., Novais, P., Neves, J., Satoh, K.: An alert mechanism for orientation systems based on Speculative computation. In: Innovations in Intelligent SysTems and Applications (INISTA), 2015 International Symposium on INnovations in Intelligent SysTems and Applications. pp. 1-8. IEEE (2015)

16. Ramos, J., Satoh, K., Novais, P., Neves, J.: Modelling an Orientation System based on Speculative Computation. In: Omatu, S., Bersini, H., Corchado, J.M., Rodríguez, S., Pawlewski, P., Bucciarelli, E. (eds.) Distributed Computing and Artificial Intelligence, 11th International Conference, Advances in Intelligent Systems and Computing, vol. 290, pp. 319-326. Springer International Publishing (2014)

17. Spitzer, R.L., Gibbon, M., Skodol, A.E., First, M.B.: DSM-IV casebook: A learning companion to the Diagnostic and Statistical Manual of Mental Disorders. American Psychiatric Association, 4 edn. (1994)

18. Ye, Y., Zheng, Y., Chen, Y., Feng, J., Xie, X.: Mining individual life pattern based on location history. In: Proceedings - IEEE International Conference on Mobile Data Management. pp. 1-10 (2009)

19. Zheng, Y.U.: Trajectory Data Mining : An Overview. ACM Trans. Intell. Syst. Technol. 6(3), 1-41 (2015) 